Programa Pesquisa para o SUS: a contribuição para gestão e serviços de saúde na Paraíba, Brasil

Research program for the Brazilian Unified Health System (SUS): the contribution to health management and services in Paraiba State, Brazil

Suely Deysny de $M$ atos Celino ${ }^{1}$

Gabriela M aria Cavalcanti Costa ${ }^{1}$

Inácia Sátiro Xavier de França ${ }^{1}$

Ednaldo Cavalcante de Araújo ${ }^{2}$

${ }^{1}$ Universidade Estadual da Paraíba. Rua Baraúnas 351 , Bairro Universitário. 58429-500 Campina Grande PB. deysny@hotmail.com ${ }^{2}$ Departamento de Enfermagem, Centro de Ciências da Saúde, UniversidadeFederal de Pernambuco.
Abstract The shared management in health of the Research Program for theU nified H ealth System (PPSUS) has the purpose of funding research in priority areas for the health of the Brazilian population. The scope of this qualitative study is to understand the researchers' perception of the contribution of research funded by the PPSUS invitations to bid in the State of Paraiba, for resolving the priority health problems of the Paraiba population, for reducing regional inequalities in health and for bolstering the management of SUS. A documentary survey of the bids and final reports of research and a semi-structured interview with 28 coordinators of these studies was conducted. Triangulation strategy of data wasused and subsequently subjected to content analysis, which converged with the categories: solving the health problems; reducing regional inequalities; contribution to management. Paraiba state needs adjustments such that the PPSUS can be fully implemented, ensuring that the knowledge generated can be converted into health policies and actions, since the research funded respond to the health needs of the population and difficulties in SUS management.

Key words Science, Health research policy, Government programs, U nified Health System
Resumo O Programa Pesquisa para o SU S: gestão compartilhada em saúde - PPSUS apresenta o intuito, entre outros, de financiar pesquisas em temas prioritários para a saúde da população brasileira. 0 objetivo deste estudo deabordagem qualitativa foi compreender a percepção dos pesquisadores sobre a contribuição das pesquisas financiadas pel os editais PPSUS no Estado da Paraíba, para a resolução dos problemas prioritários de saúdeda população paraibana, para a redução das desigualdades regi onais na área da saúde e para o fortalecimento da gestão do SU S. Realizaram-se levantamento documental nos editais e relatórios finais de pesquisas e, entrevista semiestruturada com 28 coordenadores desses estudos. U tilizou-se estratégia de triangulação de dados e estes foram submetidos à análise de conteúdo, que convergiram para as categorias: resolução dos problemas desaúde; redução das desigualdades regi onais; contribuição para a gestão. A Paraíba necessita de ajustes para queo PPSUS possa se efetivar em completude, como a garantia de que o conhecimento gerado possa se concretizar em políticas eações de saúde, visto que as pesquisas financiadas respondem às necessi dades de saúde da população e dificuldades de gestão do SU S.

Palavras-chave Ciência, Política de pesquisa em saúde, Programas governamentais, Sistema Único de Saúde 
Introdução

0 incremento do desenvolvimento científico e tecnológico dentre as competências do Sistema Único de Saúde - SU S foi incluído na Constituição Federal de 1988, em seu artigo 200. M as somente em 2004 foi aprovada a Política Nacional de Ciência, Tecnologia e Inovação em Saúde PNCTIS, parte integranteda Política Nacional de Saúde, formulada no âmbito do SUS, devendo ser pautada em seus princípios constitucionais da universalidade, integralidade e equidade, para que, por meio da produção de conhecimentos e tecnologias, possa contribuir para a redução das desigualdades sociais em saúde².

Para a superação dessas desigual dades regionais, a Política busca a articulação entre ações do governo federal, dos estados e dos municípios. Para tanto, o M inistério da Saúde, por intermé dio do Departamento de Ciência e Tecnologia da Secretaria de Ciência, Tecnologia e Insumos Estratégicos, vem desenvolvendo atividades de fomento descentralizado à pesquisa em todos os estados brasileiros, por meio do Programa Pesquisa para o SU S: Gestão Compartilhada em Saúde - PPSUS, com o propósito de financiar pesquisas em temas prioritários para a saúde da população brasileira; contribuir com o aprimoramento do Sistema Único deSaúdee; promover o desenvolvimento científico e tecnológico em saúde em todos os estados da federação ${ }^{3}$.

Sendo assim, esse Programa tem como obje tivo contribuir para o fortalecimento do esforço nacional em ciência, tecnologia e inovação em saúde, definida na PNCTIS, por meio de parcerias entre instituições públicas de fomento à pesquisa em saúde e da capacidade de gestão de ciência etecnologia nos estados', envolvendo parcerias entre a esfera federal, por intermédio do M inistério da Saúde, e a esfera estadual, pelas Fundações de Amparo à Pesquisa - FAP ${ }^{5}$. Por meio dessa iniciativa, os estados brasileiros definem prioridades de saúde em nível local, através de debates acerca de indicadores de morbi-mortalidade, além da identificação de problemas re lacionados à organização e funcionamento do sistema local de saúde ${ }^{6}$.

Especificamente, na Paraíba, os agentes executores desse programa têm sido a Fundação de A poio à Pesquisa do Estado da Paraíba - FAPESQ, e a Secretaria de Saúde do Estado. Essa parceria já viabilizou a execução de 35 projetos, nos editais publicados em 2004 e 2006, apoiando atividades de pesquisa, pela garantia de recursos financeiros que somam um montante de R\$
$990.000,00^{7}$, visando promover o desenvolvimento científico, tecnológico e/ou deinovação da área de saúde, em temas prioritários para o Estado.

A partir dessas considerações, alguns questionamentos foram elaborados acerca das pesquisas financiadas pelos editais PPSU S na Parába: quais as linhas de pesquisa prioritárias? Como as pesquisas têm contribuído para a resolução dos problemas? Como elas reduzem as desigualdades? Como fortalecem a gestão do sistema de saúde local?

Natentativadebuscar respostas para tais questões, despertou-se a necessidade decompreender a percepção dos pesquisadores sobre a contribuição das pesquisas financiadas pelos editais PPSUS na Parába para a resolução dos problemas prioritários de saúde da população, para a redução das desigual dades regionais na área de saúde e para o fortalecimento da gestão do SUS na Paraíba.

\section{Caminho metodológico}

0 estudo é de natureza exploratória, com abordagem qualitativa, sendo os sujeitos selecionados dentre os 35 pesquisadores que outrora tiveram projetos de pesquisa contemplados com financiamento dos editais PPSU S na Paraíba, nos anos 2004 (24 pesquisadores) e 2006 ( 11 pesquisadores). Foi utilizada a amostra proposital, de finida pelos seguintes critérios de inclusão: pesquisas financiadas e concluídas pelos editais PPSUS 2004 e 2006; pesquisas que os coordenadores aceitaram em participar desse estudo; acesso aos relatórios finais enviados à FAPESQ. Dessa forma, 28 coordenadores de pesquisa atenderam aos critérios definidos, sendo 17 contemplados no edital 2004 e 11 no edital 2006.

A coleta de dados foi constituída de três etapas, sendo a primeira empreendida sob os editais PPSUS 2004 e 2006; a segunda, a partir do levantamento documental nos relatórios finais das pesquisas financiadas por esses editais, disponibilizados pelaFAPESQ; eaterceira, por meio deentrevista semidirigida realizada com os coordenadores dos projetos.

Os materiais referentes aos editais e aos relatórios de pesquisa não foram analisados isoladamente, sendo associados às entrevistas gravadas e transcritas dos respectivos coordenadores das pesquisas, pela estratégia detriangulação, que pode ser utilizada em diversas situações, incluindo a utilização de uma variedade de técnicas de coleta de dados que acompanha o trabalho de investigação ${ }^{8}$. 
Para a análise dos dados, foi utilizada a técnica de análise de conteúdo, quebusca oferecer um significado aos dados, pela frequência recorrente de termos nos discursos ${ }^{9}$, optando-se pela modalidade deanálise temática. Os dados foram então, agregados em categorias que evocaram temas relacionados ao PPSU S na Paraíba, objetivo do estudo, reconhecendo que questões políticas, organizacionais e operacionais pairavam no cenário.

$\mathrm{Na}$ análise dos resultados da investigação, elencaram-se as seguintes categorias: resolução dos problemas de saúde; redução das desigualdades regionais; contribuição para a gestão.

O estudo foi aprovado pelo Comitê de Ética em pesquisa da Universidade Estadual da Paraíba, sob o Certificado de Apresentação para Apreciação Ética. Para cumprimento das exigências preceituadas pelo Conselho Nacional de Saúde, na Resolução 196/9610 , os documentos consultadose as entrevistas foram transcritos eidentificados por letras minúsculas e maiúsculas, respectivamente, em ordem alfabética, seguidas dos números 04 ou 06, indicando o ano de edital, garantido assim, o anonimato, a privacidade, e o sigilo da identidade dos atores envolvidos na pesquisa.

\section{Resultadosediscussão}

\section{Caracterização dos sujeitos}

A idade dossujeitos variou entre 35 e 60 anos, com uma média de 46,07 anos, o queindica uma população madura de pesquisadores na área de saúde. No queserefereao sexo, 11 (39,28\%) eram do sexo masculino e $17(60,72 \%)$ do feminino, contrariando pesquisa realizada por Santos et al. ${ }^{11}$ sobre o perfil dos pesquisadores brasileiros em saúde pública, em que se observou similaridade entre os gêneros

Quanto à formação básica, aponta-se a presença mais significante do profissional farmacêutico (05), seguida da formação médica (05) e do cirurgião dentista (04) , aparecendo ainda pesquisadores da área de nutrição (03), psicologia (03), biologia (02), enfermagem (02), química (01), fisioterapia (01), engenharia de alimentos (01) e antropologia (01)

Em relação à instituição a qual estavam vinculados, $19(67,85 \%)$ eram da Universidade Fe deral da Paraíba - UFPB, 05 (17,85\%) eram da Universidade Estadual da Paraíba - UEPB e 04 $(14,3 \%)$ estavam vinculados à Universidade Federal de Campina Grande - UFCG.
No que tange à titulação, apenas 05 (18,52\%) são mestres e os demais (81,48\%) doutores. Do total de mestres, três deles já estão inseridos em programas de doutorado. Vale ressaltar que 0 edital de2004 exigia titulação mínima de mestres e o edital 2006 de doutores. 0 financiamento de pesquisas de professores doutores justifica-se uma vez que o doutoramento tem como objetivo a formação de pesquisadores, que buscam 0 aprofundamento em determinado campo do saber, ao passo que o mestrado direciona-se primordialmente para o ensino, destinado para aqueles que têm o objetivo maior de lecionar ${ }^{12}$.

\section{Caracterização das pesquisas}

As pesquisas foram classificadas pelos próprios coordenadores, em relatórios enviados à FAPESQ . Sendo assim, no que se refere à natureza, 21 (75\%) foram consideradas básicas e apenas $06(21,43 \%)$ aplicadas. A penas um (01) estudo foi considerado pelo coordenador como de natureza tecnológica, por obter conhecimentos através da tecnologia de processos, no caso em questão, para a fabricação de um medicamento.

Quanto à classificação dos resultados dos estudos, os coordenadores podiam indicar uma opção e o retrato foi: sistemas de pesquisa em saúde(01), gestão e planejamento em saúde(01), políticas públicas e saúde (01), controlee participação social (02), biotecnologia (03), processo saúde-doença (10) eavaliação de políticas de saúde, programas e serviços (10). Essa classificação é orientada pelas Diretrizes Técnicas do PPSUS, que sugerem, além dessas opções, resultados de pesquisas que culminem em: regulação em saúde, trabalho em saúde, recursos humanos em saúde, insumos, informação e comunicação em saúde, bioética, avaliação de tecnologias em saúde, biossegurança e economia da saúde ${ }^{4}$.

No que tange à linha temática, no edital de 2004 todos os temas especificados foram contemplados com projetos. Dessa forma, os 17 coordenadores aprovados nesse edital abordaram os seguintes temas: saúde indígena (02), qualificação da atenção à saúde (03), gestão descentralizada do SUS (04), saúde mental (01), produção, qualidade e acesso de insumos estratégicos (03), saúde da mulher (01) e controlededoenças infecciosas e não-infecciosas (03).

Esse quadro de linhas temáticas pode estar refletindo o perfil epidemiológico do estado, visto que foram selecionados a partir de oficina de prioridades de pesquisa, como é o caso das doenças infecciosas, que havia preocupação naque- 
le ano em reduzir a morbi-mortalidade por essas causas, visto que, em 2003, ano que antecedeu o edital, estavam entre as causas mais frequentes de internação hospitalar ${ }^{13}$.

Já no edital de2006, as 11 pesquisas financiadas abordavam os temas: informação em saúde e epidemiologia (01), saúde dos portadores de necessidades especiais (01), saúde da mulher (01), alimentação enutrição (01), saúde do idoso (01), atenção básica (03), saúde, meio ambiente e doenças infecciosas (03). Interessante destacar éque os temas contemplados nesse edital já refletem algumas das prioridades pactuadas entre gestores no Pacto pela Vida, como saúde do idoso, atenção básica, doenças emergentes e endemias, alimentação e nutrição, que foram abordadas nesse documento no contexto da promoção da saúde ${ }^{14}$.

\section{Categorias temáticas}

Resolução dos problemas de saúde

A resolução dos problemas de saúde, qual seja, o enfrentamento dos principais problemas de saúde de uma população, por meio da compreensão e intervenção na esfera de seus determinantes e condicionantes constitui um dos ele mentos fundamentais na construção de uma sociedade democrática ${ }^{15}$. Dessa forma, e considerando o conceito amplo de saúde, a pesquisa em saúde deve ser guiada de maneira a promover 0 avanço científico e tecnológico, voltado para 0 desenvolvimento humano e sustentável ${ }^{3}$.

A maioria dos coordenadores das pesquisas em estudo, ao serem questionados sobre qual a contribuição destas para a resolução dos problemas prioritários de saúde da população paraibana, referiu pouca ou nenhuma contribuição nesse aspecto. N o relatório das pesquisas, também, não se verificaram informações a esse respeito.

Basicamente a gente não contribui para a re solução, a gente contribuiu para levantar problemas somente. A04

De nenhuma maneira, minha pesquisa não contribui pra isso, certo. M inha pesquisa aponta as falhas desse sistema. A06

O PPSUS sugere contribuição das pesquisas para resolução dos problemas de saúde da população. 0 que se verifica é que nas pesquisas consideradas avaliativas, seja da qualidade do atendimento, como foi o caso do sujeito A04, seja do sistema, no caso de A06, os pesquisadores não percebem que, ao mesmo tempo em que seapontam falhas, podem sugerir mudanças para a melhoria do serviço prestado à população e, assim, contribuir de alguma forma para a resolução de seusproblemas de saúde, principalmente se considerarem o conceito amplo de saúde.

Houve pesquisadores que elencaram sobre a contribuição de suas pesquisas para a resolução dos problemas de saúde da população paraibana, apontando para a orientação de ações, mesmo que indiretas, depromoção da saúde, apesar dessa preocupação não ter sido descrita no relatório.

Alertar e esclarecer a população acerca da fragilidade do controle de qualidade dos chás comercializados em sachês em nosso país. G04

Olha só, a pesquisa ela tem seus dados. Com a divul gação desses dadoséque ela tem como efetivamente contribuir. K06

A Carta de Otawa ${ }^{16}$ define cinco campos de ação da promoção da saúde: elaboração e imple mentação de políticas públicas saudáveis; criação de ambientes favoráveis à saúde; reforço da ação comunitária; desenvolvimento dehabilidadespessoais e; reorientação dos serviços de saúde.

0 desenvolvimento de habilidades pessoais como ação de promoção da saúde foi citado nas falas G04 e K06, visto que se referem ao esclarecimento da população pela divulgação dos resultados para a sociedade. Ainda segundo a Carta de Otawa16, a divulgação de informação, educação para a saúde e intensificação das habilidades vitais aumentam as opções disponíveis para que a sociedade possa exercer maior controle sobre sua própria saúde e sobre o meio-ambiente, além de estar apta a fazer opções que conduzam a uma saúde melhor.

Os coordenadores também observaram a perspectiva de tratamento de baixo custo como contribuição para a resolução dos problemas de saúde da população paraibana:

Daí a importância que achamos desse estudo, descobrir novas moléculas que possam aumentar o plantel farmacêutico decombatea Leishmania. E06

Seria talvez um tratamento de baixo custo, favorecendo a população de baixa renda, já que as substâncias são extraídas de vegetais da região. F06

Os dados encontrados nos relatórios desses coordenadores são congruentes com suas falas, ao reportarem a perspectiva de tratamento acessível à população:

A procura contínua por novos agentes terapêuticos para as leishmanioses ainda é essencial. U ma alternativa pertinente seria a utilização de compostos vegetais isolados que podem fornecer novos model os terapêuticos no tratamento deleishmanioses. e06

U ma vez comprovada a atividadeinibidora dos derivados leishmanicidas, a emergência do desen- 
volvimento de um novo fármaco com visíveis vantagens econômicas seria prontamente aplicável, em especial ao facilitar o acesso do paciente ao medicamento. f06

Em relação à descrição de f06, verifica-se a preocupação do pesquisador com a descoberta de tratamento mais eficaz e de menor custo para o problema da leishmaniose, doença negligenciada no país que apresenta um tratamento com medicamentos de el evada toxicidade, risco de re sistência eal to custo. A leishmaniose, assim como as outras doenças negligenciadas, tem sido considerada prioridade para pesquisas pela Secretaria deCiência, Tecnologia el nsumosEstratégicos, que financiou em 2004, por meio de um edital, 33 pesquisassobre dengue. Em 2006, a partir deum rico processo de definição de prioridades depesquisa, direcionou-se mais de $\mathrm{R} \$ 20$ milhões para o financiamento de projetos em malária, doença de Chagas, dengue, leishmaniose, tuberculoseehanseníase $^{17}$. Esse edital foi publicado em âmbito nacional, no qual poderiam concorrer pesquisadores de todo o país, não sendo encontrada proposta de financiamento de pesquisas específicas para essa área na instância estadual.

Redução das desigualdades regionais

0 território brasileiro é caracterizado por grandes desigual dades socioeconômicas que aparecem como um dos traços mais marcantes da sociedadee, seentendidas do ponto de vista regional, se constituem em alvo de preocupação e de políticas públicas ${ }^{18}$, devido às disparidades de desenvolvimento existentes entreas grandes macrorregiões brasileiras.

O PPSUS foi idealizado com o propósito de contribuir para a redução das desigualdades regionais no campo da ciência, tecnologia einovação em saúde. Apesar disso, grande parte dos coordenadores de pesquisas financiadas por esse programa, ao serem questionados sobre qual a contribuição dos seus estudos para diminuir essas desigualdades, afirmou não ter focado esse aspecto.

Com relação à contribuição para as desigualdades na questão desaúde, eu acho queo resultado do trabalho poderia ajudar muito isso aí, colaboraria muito sealguma medida fosse ou tivesse sido tomada eisso eu não posso informar. $J 04$

Dejeito nenhum! Não épesquisa quevai reduzir desigualdade. A06

Nenhum desses sujeitos fez qualquer observação a respeito desse assunto em relatório, já sendo esperado que eles também não apresentassem nenhuma contribuição na entrevista. Tal- vez ainda faltea consciência crítica dos pesquisadores em refletir sobre a contribuição de suas pesquisas para o desenvolvimento da sociedade como um todo, o que leva a análises equivocadas quando se afirma que pesquisa não pode reduzir desigualdade, como é o caso do sujeito A06. Essa fala mostra indício de que os conhecimentos produzidos cientificamente se apóiam muito nos interesses do próprio pesquisador que os produzem, para obter reconhecimento da comunidade científica, ficando a responsabilidade social na produção e divulgação do conhecimento, por vezes, em posição secundária ${ }^{19}$.

A superação das desigualdades regionais em ciência e tecnologia em saúde como objetivo do PPSU S atende às estratégias da Política Nacional deCiência, Tecnologia e I novação em Saúde, que tem como ponto central contribuir para o desenvolvimento nacional sustentável e para a produção de conhecimentos técnicos e científicos ajustados às necessidades econômicas, sociais, culturais e políticas do país².

H ouve aqueles sujeitos que, quando abordados acerca da contribuição dos seus estudos para reduzir as desigualdades regionais em saúde, apresentaram falas acerca de que as pesquisas contribuíram apenas para o grupo estudado, entendendo que estas puderam favorecer a redução das desigualdades existentes entre a população em estudo e os demais grupos populacionais, mesmo dentro da mesma região.

Os pacientes atendidos na pesquisa tiveram acesso aos meios diagnósticos e tratamento sem se submeterem às longas filas do SU S. B04

Ela podeter colaborado indiretamente, porque as gestantes que participaram do projeto tiveram uma assistência mais de perto. $J 06$

$\mathrm{N}$ ão foram constatados dados sobre esse aspecto nos relatórios. A pesar disso, o que as falas anteriores indicam é que os estudos, mesmo não tendo ampla contribuição para a população da região, puderam favorecer de alguma forma 0 grupo estudado, seja pelo acesso a atendimento mais rápido, como referido, pelo sujeito B04, ou a assistência de melhor qualidade, como sugere J06.

Alguns sujeitos exaltaram a contribuição de seus estudos para diminuir as desigualdades regionais no campo da saúde, oferecendo, a partir da aplicação dos resultados, possibilidade de maior resolutividade dos serviços para os problemas de saúde da população.

eu acho que melhorou a questão do acesso aos serviços da média complexidade, a população tinha grande dificuldade. 104 
Q uando a gente aponta maneiras de como re solver, faz com que o estado como um todo cresça em termos de ter uma saúde pública resolutiva, forte, queimpacte que pensenuma lógica do usuário. $\mathrm{CO6}$

As falas acima permitem a compreensão que as pesquisas têm procurado contribuir no sentido de apontar caminhos para a resolutividade de determinados problemas de saúde da população, apesar de esse aspecto não ter sido levantado em relatório. Os coordenadores fazem referência à resolutividade caracterizando-a por ações de saúde, condições dos serviços e do sistema de saúde, que resultem em efeito positivo para a população, ou seja, em um atendimento qualificado.

Entendendo esse conceito de forma ampla, a resolutividadee seus efeitos devem ser capazes de transformar positivamente a situação de saúde deum indivíduo ou deum grupo, presumindo a possibilidade de avaliação das ações praticadas, bem como o conhecimento e análise dos seus resultados. N esse sentido, o objetivo passa a ser saber não só até que ponto a resolutividade está sendo alcançada na prestação de serviços, mas conhecer quais modificações estão ocorrendo nas situações-problema apresentadas, e assim, priorizar ações de saú de e estabel ecer condições cada vez mais resolutivas para os serviços ${ }^{19,20}$.

Os pesquisadores ainda acreditam que suas pesquisas têm contribuído para reduzir as desigual dades regionais em saúde, ao favorecer a qualificação de recursos humanos para a pesquisa.

Eu acho que ela colaborou para a capacitação de profissionais de recursos humanos. D04

Se a gente pensa de uma forma ampla de combate às desi gualdades, seria a formação de pessoal, a gente formou mestre, formou graduados e isso realmente eu imagino que é uma forma de, talvez pequena, combater as desigualdades. E06

M esmo esse aspecto não tendo sido abordado nos relatórios de nenhum dos sujeitos, as falas refletem o importantepapel do PPSU S na Paraíba na formação de pesquisadores em saúde no estado, que consequentemente, aumentaria também a quantidade de pesquisas que possam contribuir para as necessidades de saúde da Paraíba.

Esse programa deve estar em todo o território brasileiro, em consonância com o que preconiza a Organização M undial de Saúde, contribuindo para a superação das desigualdades regionais, pela formação de recursos humanos para a pesquisa, além da inserção dessas nas agendas das secretarias estaduais de saúde ${ }^{3}$.

A formação, a capacitação e a absorção de recursos humanos constituem uma das princi- pais estratégias da Política Nacional de Ciência, Tecnologia e Inovação em Saúde, que se faz essencial para desenvolver a produção do conhecimento científico e tecnológico nos programas, ações e serviços de saúde e aperfeiçoar a gestão deciência, tecnologia einovação em saúde².

\section{Contribuição para a gestão}

Com o objetivo de contribuir para o fortale cimento da gestão da saú de e para a resolução de problemas prioritários de saúde da população brasileira, o M inistério da Saúde apoia financeiramente o desenvolvimento de pesquisas, por intermédio do PPPSUS4. N esse sentido, os pesquisadores foram questionados sobre a contribuição de seus estudos para a gestão dos serviços de saúde, fornecendo algumas respostas que refletiram a pouca preocupação desses com a devolução dos resultados à gestão:

$\mathrm{N}$ ão sei se efetivamente houve essa contribuição. $\int 04$

Eu acho queem termo degestão, de gestão dire tamente, eu não teria dados, porque a gestão trabalha muito com dados. $\mathrm{H} 06$

Embora os coordenadores tenham relatado em entrevista a não aplicabilidade de seus estudos para a gestão, deve-se considerar que o pesquisador tem um compromisso social com seus estudos, principalmente no que se refere a projetos financiados pelo PPSUS, que tem como objetivo contribuir com a política desaúde local. Além disso, as falas dos pesquisadores divergem com os descritosem relatórios, quando colocam quesuas pesquisas trazem contribuições para a gestão:

0 delineamento do quadro desaúdebucal indígena servirá para embasar e orientar o planejamento ea demanda por atenção à saúde bucal nos planos preventivo, educativo e curativo do SU S. j04

Acreditamos que o gestor possa criar ou fortalecer programas de tratamento multi e interdisciplinares que enfoquem ações educativas na promoção à saúde, voltados para a melhoria da qualidade de vida. h06

Essas ações, ou mesmo propostas expressas nos relatórios, que certamente não foram efetivadas, pois não houve articulação com a gestão para sugerir essas ações propostas no relatório, podem ter sido apresentadas apenas como formalidade exigida no formulário padrão de apresentação do seminário, e até mesmo no projeto, no qual o coordenador já teria que apontar a aplicabilidade de sua pesquisa para o Sistema Único de Saúde.

Alguns pesquisadores elencaram como contribuição das pesquisas em questão para a ges- 
tão dos serviços de saúde a proposição de políticas e programas:

Eu acho que a contribuição do estudo seria ajudar justamentena política de promoção da saúde. $\mathrm{H} 06$

É possível contribuir com a gestão elaborando estratégia de intervenção que possa ser realizada [...] Por isso os resultados devem ser enviados para o M inistério da Saúde, para o pessoal da FAPESQ, para a universidade, a prefeitura, o governo. 106

Asfalas, ao serem confrontadas com o documentado nos relatórios das pesquisas, apontaram congruência de informações no caso do sujeito $\mathrm{H} 06$ :

Acreditamos que o gestor possa criar ou fortalecer programas de tratamento multi-interdisciplinares que enfoquem ações educativas na promoção à saúde, voltados para a mel horia da qualidade de vida. h06

No caso do sujeito 106, a pesquisa não foi divulgada à gestão local, conforme relatado em seu discurso, também não fica clara sua contribuição, nem na entrevista, nem no relatório.

É possível sim que o gestor possa desenvolver programas baseados nos resultados de pesquisas, desde queseapropriem dos conhecimentos produzidos nos estudos. As Fundações de Amparo à Pesquisa também têm a responsabilidade de apresentar ao M inistério da Saúde uma análise dos resultados das pesquisas, descrevendo o potencial de incorporação desses no sistema e serviços de saúdet.

As políticas públicas são princípios que norteiam a ação do poder público, diretrizes, regras e procedimentos para as relações entre o poder público e a sociedade, sendo explicitadas, sistematizadas ou formuladas em documentos (leis, programas) que orientam ações que geralmente envolvem aplicações de recursos públicos ${ }^{21}$. No caso da saúde, as políticas públicas se materializam na forma de programas de atenção à saúde, que são implementados pelos gestores municipais, estaduais ou federal, com o objetivo de melhorar a qualidade da assistência à saúde da população.

A avaliação do sistema/serviços de saú detambém foi mencionada pelos coordenadores como contribuição para a gestão, tendo como objetivo principal a produção da informação que contribua para o aprimoramento do objeto avaliado:

Eu acho que, ao avaliar esses dados, podemos perceber a imperfeição, a fragilidade ou essa insuficiência. K04

Eu acho que serviu para avaliar o serviço que estava sendo destinado àquela população. 104
O sujeito K04 descreve em relatório estratégias de ações que parece não terem sido realizadas, indo muito mais além da avaliação do sistema, colocando a importância de constituir uma estratégia de acompanhamento das políticas públicas de saúde no estado da Paraíba, principalmente aquelas voltadas à saúde mental:

Tal estratégia se materializará através de um observatório de políticas públicas em saúde, a ser instituído na UFCG. k04

No caso do sujeito 104, a fala da entrevista é congruente com o documentado em relatório:

As informações apresentadas mostram uma realidade preocupante, a qual exigirá grandes desafios para a superação, no sentindo de melhores indicadores de saúde bucal. i04

A avaliação em saúde tem como pressuposto a avaliação da eficiência, eficácia e efetividade das estruturas, processos e resultados relacionados ao risco, acesso e satisfação dos cidadãos frente aosserviços públicos desaúde na busca da resolutividadeequalidade, sendo partefundamental no planejamento e na gestão do sistema de saúde ${ }^{22}$.

Nesse sentido, apesar de se tratarem de pesquisas avaliativas, tudo leva a crer que não contribuíram para a resolutividade dos problemas questionados ou para a melhoria da qualidade dos serviços em questão, conforme descrito nos relatórios.

Alguns coordenadores contemplaram a meIhoria da qualidade dos serviços de saúde como contribuição de suas pesquisas para a gestão:

[...] planejar outras ações de acordo com as necessidades detectadas pela pesquisa. 104

A gente espera conseguir que, as pessoas com deficiência tenham acesso a um maior número ea melhores serviços de atenção de média complexidade e reabilitação. K06

Ao confrontar esses dados com os relatórios, constata-se congruência das informações, apontando as perspectivas de melhoria da prestação dos serviços a partir das informações obtidas através de suas pesquisas:

Os indicadores foram utilizados para identificação de prioridades e planejamento das ações de saúde bucal nos três polos base do Distrito Sanitário Especial Indígena da Paraíba. i04

Oferecer ferramentas para que os gestores de saúde e as entidades de defesa dos direitos das pessoas com deficiência possam conseguir uma meIhora dos serviços de saúde voltados para esta população. k06

Um serviço pode ser considerado de qualidade quando se consegue o padrão mais elevado de desempenho ${ }^{23}$, atendendo às exigências dos clien- 
tes. A qualidade em atenção à saúde envolve múltiplos aspectos, mas de forma geral, esperase que os serviços atendam às necessidades de saúde definidas tecnicamente, contemplando o amplo espectro das suas atribuições, quais sejam, a promoção, a prevenção, a assistência e a reabilitação $0^{24}$.

Dessa forma, persiste o desafio de organizar estudos e pesquisas que contemplem, não só ações de promoção da saúde, como também estratégias de melhoria da qualidade dos serviços prestados à população. A pesar de os coordenadores terem apresentado em entrevistas e relatórios essa possibilidade, parece que não foram colocadas em prática, visto que esses resultados não foram discutidos com a gestão.

\section{Considerações finais}

Verificaram-se discursos de coordenadores de pesquisas que se expressaram negativamente no quese refereà colaboração de suas pesquisas para a resolução dos problemas de saúde da população paraibana, sendo esse aspecto também pouco apresentado em relatório. Quando referida, essa contribuição se deu através de proposição de ações de promoção da saúde e perspectiva de tratamento de baixo custo, para aqueles estudos que tratavam da descoberta de novas drogas terapêuticas para o tratamento de doenças negligenciadas. Essas ações ainda estiveram limitadas à incorporação dos resultados pela gestão.

Todo o esforço do M inistério da Saúde, através do Decit, com a efetivação do PPSUS, tem sido principalmente no sentido de tentar reduzir as desigualdades regionais desaúde através das ações de fomento, que não foi abordado em nenhum dos relatórios apresentados. Surgiram declarações de contribuições relativas apenas ao grupo estudado, além da qualificação de recursos hu- manos, aspecto bastante importante em se tratando da redução de desigualdades relacionadas ao número de pesquisadores em diferentes regiões e, consequentemente, à desigualdade de investimentos em pesquisas nas regiões brasileiras.

Foram constatadas falas dos pesquisadores que não relataram contribuição das suas pesquisas para o fortalecimento da gestão dos serviços de saúde. Os que o fizeram discorreram sobre ações relativas à proposição de políticas e programas, avaliação do sistema/serviços de saúde, perspectiva de desenvolvimento de novas drogas e melhoria da qualidade dos serviços. M esmo esses, não apresentaram contribuição concreta, visto que essas ações dependeriam da divulgação desses resultados à gestão, o que parece ter acontecido somente através de Seminário de Avaliação, havendo ainda, uma imensa dificuldade para que as propostas cheguem aos serviços de saúde.

A pesar disso, entende-se que o PPSUS pode verdadeiramente contribuir para o desenvolvimento científico etecnológico no país, através da redução das desigualdades regionais nesse campo, embora na Paraíba ainda necessite de alguns ajustes para que efetivamenteesse programa possa seconcretizar em completude, como, por exemplo, agarantia dequeo conhecimento gerado possa se concretizar em políticas e ações de saúde, visto que as pesquisas financiadas no estado respondem às necessidades de saúde da população, e até mesmo às dificuldades de gestão do SUS.

Por fim, o estudo indicou a necessidade dos pesquisadores compreenderem o propósito dos editaisPPSUS, participarem efetivamentedosse minários juntamente com os gestores das instâncias e, assim, fortalecer o papel da pesquisa na resolutividade dos problemas de saúde, como prática de interesse recíproco e de solidariedade, para que esse programa verdadeiramente possa colaborar com a redução das desigualdades regionais em saúde. 


\section{Colaboradores}

SDM Celino, GM C Costa, ISX de França eEC de Araújo são igualmente responsáveis pela definição do objeto de estudo, da abordagem metodológica, pesquisa bibliográfica, elaboração e aplicação do instrumento, análise dos dados, redação, revisão do artigo e aprovação para publicação.

\section{Referências}

1. Brasil. Constituição 1988. Constituição da República Federativa do Brasil, 1988. Braślia: Senado Federal, Secretaria Especial de Editoração e Publicações; 2005.

2. Brasil. M inistério da Saúde (MS). Secretaria de Ciência, Tecnologia e Insumos Estratégicos. Departamento de Ciência e Tecnologia. Política Nacional de Ciência, Tecnologia e Inovação em Saúde. 2ª Edição. Brasília: M S; 2006.

3. Brasil. M inistério da Saúde (M S). Secretaria de Ciência, Tecnologia e Insumos Estratégicos. Departamento de Ciência e Tecnologia. Seleção de prioridades de pesquisa em saúde: guia PPSUS. 2a Edição. Brasília: M S; 2009.

4. Brasil. Ministério da Saúde (MS). Secretaria de Ciência, Tecnologia e Insumos Estratégicos. Departamento de Ciência e Tecnologia. Programa Pesquisa para o SU S: gestão compartilhada em saúde (PPSUS) - diretrizes técnicas. Brasília: M S; 2007.

5. Conselho Nacional de Secretários de Saúde (Brasil). Ciência e Tecnologia em Saúde. Coleção progestores - para entender a gestão do SU S. Brasília: CONASS; 2007.

6. Guimarães R. Bases para uma política nacional de ciência, tecnologia e inovação em saúde. Cien Saude Colet 2004; $9(2): 375-387$.

7. Fundação de Apoio à Pesquisa do Estado da Paraíba (FAPESQ). Edital 002/2009 - PPSUS/FAPESQ. [acessado 2009 set 02]. Disponível em: http://www. fapesq.rpp.br/PPSU SFAPESQEDITAL2009.pdf.

8. M inayo MCS, Assis SG, Souza ER, organizadores. Avaliação por triangulação de métodos: abordagem de programas sociais. Rio de Janeiro: Fiocruz; 2005.

9. Turato ER. Tratado de metodologia da pesquisa clínico-qualitativa. 3a Edição. Rio de Janeiro: Vozes; 2008.

10. Brasil. Ministério da Saúde. Conselho Nacional de Saúde. Resolução no. 196 de 10 de outubro de 1996. Diretrizes e Normas Regulamentadoras de Pesquisas Envolvendo Seres Humanos. Diário O ficial da União 1996; out 16.

11. Santos SM C, Lima LS, M artelli DRB, M artelli-Júnior $H$. Perfil dos pesquisadores da saúde coletiva no Conselho Nacional de Desenvolvimento Científico e Tecnológico. Physis 2009; 19(3):761-775.

12. Garcia FG, Eid Júnior W. Como planejar a educação. São Paulo: Publifolha; 2001.

13. Brasil. Ministério da Saúde (M S). DATASUS. Informações de Saúde. Morbidade hospitalar do SUS Paraíba. Internações segundo lista morb CID-10 dez 2003. [acessado 2011 jan 25]. Disponível em: http:// tabnet.datasus.gov.br/cgi/tabcgi.exe?sih/cnv/mipb. def

14. Brasil. Ministério da Saúde (M S). Secretaria Executiva. Departamento de Apoio à Descentralização/ Coordenação Geral de Apoio à Gestão Descentralizada. Diretrizes operacionais dos Pactos pela Vida, em Defesa do SUS e de Gestão. Brasília: M S; 2006.

15. Batistela C. Análise da situação de saúde - principais problemas de saúde da população brasileira. In: Fonseca AF, Corbo AM DA, organizadoras. 0 território e o processo saúde-doença. Rio de Janeiro: EPSJV, Fiocruz; 2007. p. 121-58. 
16. Carta de Otawa. Primeira Conferência Internacional sobre Promoção da Saúde. Otawa, nov 1986. [acessado 2010 ago 18]. Disponível em: http://www. opas.org.br/promocao/uploadArq/Ottawa.pdf

17. Brasil. M inistério da Saúde (M S). Secretaria de Ciência, Tecnologia e Insumos Estratégicos. Departamento de Ciência e Tecnologia. Ciência, tecnologia e inovação em saúde. Brasília: M S; 2008.

18. Barros FAF. Os desequilíbrios regionais da produção técnico-científica. São Paulo em Perspectiva 2000; 14(3):12-19.

19. Sacardo MS, Hayashi MCPI. Quem dita os "rumos" das publicações científicas originadas de dissertações e teses? reflexão para a área da educação física. Rev Conexões 2007; 5(1):42-50.

20. Degani VC. A resolutividade dos problemas de saúde: opinião de usuários em uma unidade básica de saúde [dissertação]. Porto Alegre (RS): Universidade Federal do Rio Grande do Sul; 2002.

21. Teixeira EC. O papel das políticas públicas no desenvolvimento local e transformação da realidade. Associação dos Advogados de Trabalhadores Rurais da Bahia 2002. [acessado 2010 dez 27]. Disponível em: http://www.dhnet.org.br/dados/cursos/ aatr2 /a_pdf/03_aatr_pp_papel.pdf , 2002

22. Brasil. M inistério da Saúde (MS). Secretaria de Atenção à Saúde. Departamento de Regulação, Avaliação e Controle de Sistemas. Programa nacional de avaliação de serviços de saúde. Brasília: M S; 2004.

23. Maximiano ACA. Teoria geral da administração. 6a Edição. São Paulo: Atlas; 2007.

24. Campos CEA. Estratégias de avaliação e melhoria contínua da qualidade no contexto da atenção primária à saúde. Rev Bras Saude M atern Infant 2005; 5(Supl. 1):63-69.

Artigo apresentado em 13/03/2011 Aprovado em 01/04/2011

Versão final apresentada em 10/04/2011 